\title{
ANÁLISIS CIENCIOMÉTRICO \\ DE MAYOR IMPACTO ACERCA \\ DEL DUELO Y LA PÉRDIDA EN PERSONAS CON DISCAPACIDAD INTELECTUAL
}

\section{Scientometric Analysis with the Greatest Impact on Grief and Bereavement of People with Intellectual Disability}

Nazaret Martínez Heredia

Universidad de Granada. Facultad de Ciencias de la Educación. Departamento de Pedagogía nazareth@ugr.es

Recepción: 6 de agosto de 2019

Aceptación definitiva: 16 de abril de 2020

Resumen: El duelo puede definirse como el conjunto de procesos psicológicos y psicosociales que siguen a la pérdida de un ser querido con el que la persona en duelo estaba psicológicamente vinculada, tratándose de una reacción comportamental y emocional causada por la ruptura del vínculo afectivo y manifestándose en forma de sufrimiento. El presente estudio pretende analizar cienciométricamente la producción científica de mayor impacto sobre el duelo y la pérdida en personas con discapacidad intelectual en las principales bases de datos de carácter multidisciplinar: Scopus y Web of Science (WoS). Para ello se delimitaron tres descriptores: "grief", "bereavement" e "intellectual disability”. El procedimiento se realizó mediante una búsqueda combinada a tres, analizando la información en torno a ocho variables: publicaciones entre los años 2009-2019, número de registros en la producción científica desde los años 2009 a 2019, número de registros por tipología de documentos publicados, número de registros por títulos de las fuentes procedentes de la literatura científica de mayor impacto, número de registros por países, número de registros por instituciones, número de registros por autores y número de citas en artículos. Los resultados muestran que nos encontramos con un campo científico en vías de desarrollo. Es importante trabajar en investigaciones para conocer de manera detallada las características en el proceso de duelo, junto a una herramienta de uso profesional que ayude a determinar las necesidades de apoyo de las personas con discapacidad intelectual.

Palabras Clave: duelo; pérdida; análisis bibliométrico; discapacidad intelectual. 
АвsтRAст: Grief can be defined as the set of psychological and psychosocial processes that follow the loss of a loved one with whom the grieving person was psychologically linked, being a behavioral and emotional reaction caused by the breakdown of the emotional bond manifesting itself in a way of suffering. The present study intends to scientifically analyze the scientific production with the greatest impact on grief and bereavement of people with intellectual disabilities in the main multidisciplinary databases: Scopus and Web of Science (WoS), for this purpose three descriptors were delimited: "grief", "bereavement" and "intellectual disability". The procedure was carried out by means of a combined search by triplets, analyzing the information around eight variables: publications between the years 2009-2019, number of records in the scientific production from the years 2009 to 2019, number of records by type of documents published, number of records by titles of the sources from the scientific literature, number of records by countries, number of records by institutions, number of records by authors and number of citations in articles. The results show that we find a scientific field in development. It is important to work in research to know in detail the characteristics in the grieving process, together with a professional use tool that helps determine the support needs of people with intellectual disabilities.

KEY WORDS: grief; bereavement; bibliometric analysis; intellectual disability.

\section{Introducción}

$\mathrm{H}$ ASTA HACE MUY POCO TIEMPo, las personas con discapacidad intelectual no solían vivir más tiempo que sus padres, existiendo altas tasas de mortalidad precoz; actualmente la situación ha cambiado, la esperanza de vida ha aumentado para toda la población, incluidas las personas con discapacidad intelectual (Muñiz, Alcedo y Gómez, 2017). Por esta razón es sumamente importante hablar del duelo y de la pérdida en personas con discapacidad intelectual, pero, previamente, debemos tener claro qué es el duelo y qué puede provocar.

La pérdida de un ser querido y, en general, todas las pérdidas afectivas despiertan en la persona un conjunto de reacciones afectivas, conductuales y cognitivas que llamamos duelo; y en el caso de las personas con discapacidad intelectual, atraviesan por distintas etapas, respondiendo a la muerte en la mayoría de los casos de una índole similar a como lo hace la población en general, tristeza, ansiedad, angustia, llanto, hiperactividad y comentarios inapropiados (McRitchie et al., 2014). Numerosos estudios ponen de manifiesto que su proceso de duelo se caracteriza por experiencias similares a las analizadas en otras poblaciones (Brickell y Munir, 2008). Haciendo referencia a la salud mental, el duelo pone en cuestión la adaptación de la persona a nivel psicológico, físico, social y biológico, por lo que estudiar las implicaciones que posee es de especial relevancia, implicaciones que varían según la etapa por la que atraviesa la persona (Solana, 2005), junto al nivel de discapacidad intelectual y de experiencias previas (James, 1995).

Worden (2010) lo define como un proceso activo en el cual se dan una serie de tareas que le proporcionan a la persona una sensación de esperanza y fuerza para poder seguir su vida sin someterse al estrés de reconocer si lo está haciendo de manera 
positiva o negativa. Se trata de un conjunto de conductas y representaciones mentales vinculadas a una pérdida. Es una experiencia emocional de la pérdida que no solo se manifiesta a través de formas conductuales o sentimientos, sino también culturales e institucionales que pueden adoptarse a través del luto. Cada cultura utiliza diferentes estrategias de afrontamiento para enfrentarse a las consecuencias de una pérdida (Moorley, Cahill y Corcoran, 2016). Poch y Herrero (2003) hablan de las características que debe tener un proceso de duelo, siendo estas: se trata de un proceso que evoluciona a través del tiempo y del espacio; es normal reaccionar ante una pérdida; dinámico; depende del reconocimiento social; y no solo se trata de un proceso individual, sino también colectivo y activo. Robles y Medina (2002) determinan que el apoyo social refuerza lazos sociales que ayudan a la persona a reconocer su nuevo rol producido ante la pérdida cuando existen disturbios psicológicos. Campbell y Bell (2010) evidencian que en personas con discapacidad intelectual, si se les proporciona el apoyo necesario, el duelo puede ser menos intenso o incluso cesar en poco tiempo. Otros autores, como Bucay (2006), lo concretan como un proceso doloroso de elaboración de una pérdida, inclinado a la armonización interna y externa del sujeto frente a una nueva realidad, comprendiendo una serie de reacciones intelectuales, físicas, emocionales, conductuales y espirituales producidas por esa muerte, llegando incluso a modificar el estilo de vida de dichas personas que lo sienten. González Romero y López (2013) entienden que el duelo se caracteriza por ser una vivencia personal, intransferible y dinámica sin conocer cuándo comienza o termina dicho proceso. Existen tres factores implicados en el duelo de esta población: los derivados de la discapacidad intelectual, el impacto de la discapacidad en el apego y los factores ambientales, siendo la discapacidad intelectual la que se relaciona de forma directa con la comprensión del concepto de muerte (Blackman, 2016).

Se trata de un proceso normal en el que afloran diferentes sentimientos, por ejemplo, rabia, dolor, culpa y tensión, ya que el duelo normal es la reacción de adaptación a cualquier tipo de pérdida, implicando una sucesión de emociones y sentimientos que precisan de un cierto tiempo para ser superados (Bild y Gómez, 2008). Shear (2012) expone que el proceso de duelo en personas con discapacidad intelectual debe entenderse como un proceso emocional intenso, que provoca dolor y angustia, pero no se trata de una enfermedad ni un trastorno ansioso-depresivo. Por lo tanto, ayudarle a entender el concepto de muerte ayudaría a comprender mejor las consecuencias, los sentimientos y los procesos involucrados en el duelo, junto a la facilitación de apoyos adecuados para evitar la cronificación del mismo (Rodríguez Herrero, Izuzquiza y Herrán, 2015).

El duelo no se trata de un estado ni de un momento, se trata de un proceso que puede durar entre dos y cinco años mediante el cual las personas pueden aceptar una pérdida y volver a reorganizar su propia vida. Las vivencias, las acciones y los sentimientos del sujeto en duelo varían a lo largo de los años desde la desorganización emocional y estupor hasta la reorganización emocional y recuperación de proyectos vitales e intereses. Todas estas características del proceso de duelo permiten concluir que, aunque las personas con discapacidad intelectual pueden presentar un curso no patológico, existe una mayor vulnerabilidad y un mayor riesgo de sufrir una cronificación del mismo (Fernández, Alcedo y Gómez, 2018). 
Debido a la importancia de la investigación en relación a esta temática en personas con discapacidad intelectual, la presente investigación tiene como objetivo principal analizar cienciométricamente la producción científica de mayor impacto sobre el duelo y la pérdida en personas con discapacidad intelectual en las principales bases de datos de carácter multidisciplinar: Scopus y Web of Science (WoS).

\section{Metodología}

Para la realización del análisis de los metadatos de la producción científica de mayor impacto se ha utilizado una metodología propia de los estudios cienciométricos, cuyo objetivo se centra en describir de manera cuantitativa la información obtenida (Pertegal-Vega, Oliva-Delgado y Rodríguez-Meirinhos, 2019). Para atender al objetivo de investigación, se delimitaron tres descriptores indexados en ERIC Thesaurus para la búsqueda en las bases de datos: "duelo-bereavement", "discapacidad intelectual-intellectual disability" y "pérdida-grief”. La elección de estos descriptores se centra principalmente en la posibilidad de aglutinar la temática objeto de estudio, teniendo en cuenta que duelo, pérdida y discapacidad intelectual son los ejes centrales de nuestra investigación. El procedimiento de búsqueda se realizó a través de combinaciones a tres limitando el campo a la aparición de las mismas en el título, resumen y tesauros dentro del área de ciencias sociales.

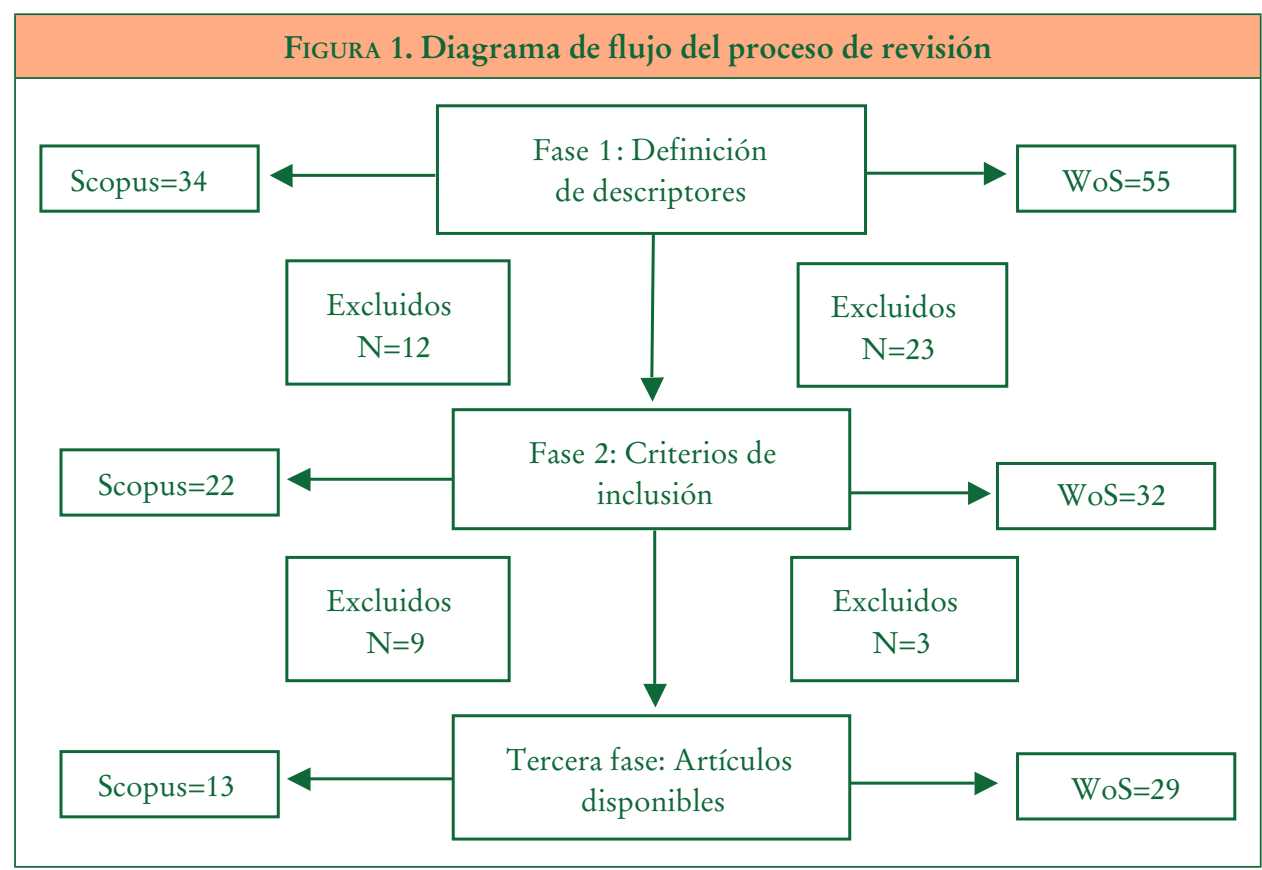

Ediciones Universidad de Salamanca / CC BY-NC-ND

Siglo Cero, vol. 51 (3), 2020, julio-septiembre, pp. 47-60 
Las bases de datos han sido las colecciones principales de WoS (Web of Science) y Scopus, ya que ambas bases agrupan la producción científica de mayor impacto. La variable estadística estudiada fue la producción científica de mayor impacto a través de un muestreo estadístico longitudinal comprendido entre los años 2009-2019. Para ello, se siguieron los siguientes procesos: conocer diferentes aspectos sobre el duelo y la pérdida en personas con discapacidad intelectual; y definir los indicadores bibliométricos que fueron utilizados para el análisis de la producción científica acerca de la temática a investigar seleccionando y analizando los datos obtenidos a partir de dicho análisis. La recogida de datos tuvo lugar en julio de 2019. La muestra final de referencias ha sido de $42(\mathrm{n}=42)$, de las cuales 13 pertenecen a la base de datos Scopus y 29 a la base de datos Web of Science. En la Figura 1 podemos ver el proceso seguido hasta la obtención de la muestra final.

Una vez establecida la muestra final, para el análisis de la información de cada variable se utilizó el software de análisis cuantitativo SPSS para Windows versión 25. Los metadatos obtenidos se han seleccionado a través de las siguientes variables consideradas en numerosas investigaciones relacionadas con nuestro objeto de estudio (Corrales-Reyes, Fornaris-Cedeno y Reyes-Pérez, 2018; Salgado-Orellana, Díaz-Levicoy, Ferrada y Parraguez, 2019; Rodríguez y Gallego, 2019; Fuentes y Ortega, 2019):
a) V1. Publicaciones entre los años 2009-2019.
b) V2. Número de registros en la producción científica desde los años 2009 a 2019.
c) V3. Número de registros por tipología de documentos publicados.
d) V4. Número de registros por títulos de las fuentes procedentes de la literatura científica de mayor impacto.
e) V5. Número de registros por países.
f) V6. Número de registros por instituciones.
g) V7. Número de registros por autores.
h) V8. Número de citas en artículos.

\section{Resultados}

El análisis de los artículos científicos ha permitido extraer los siguientes resultados, siendo estos:

\subsection{V1. Publicaciones entre los años 2009-2019}

En relación al número de publicaciones publicadas durante los últimos diez años (2009-2019), en la base de datos Scopus ( $\mathrm{n}=13)$ solamente un 7,69 \% se encuentra en acceso abierto $(\mathrm{n}=1)$ y en la Web of Science $(\mathrm{n}=29)$ un $13,79 \%(\mathrm{n}=4)$. Por lo que en la segunda base la documentación científica de acceso abierto es superior. 
Figura 2. Porcentaje de publicaciones entre los años 2009-2019

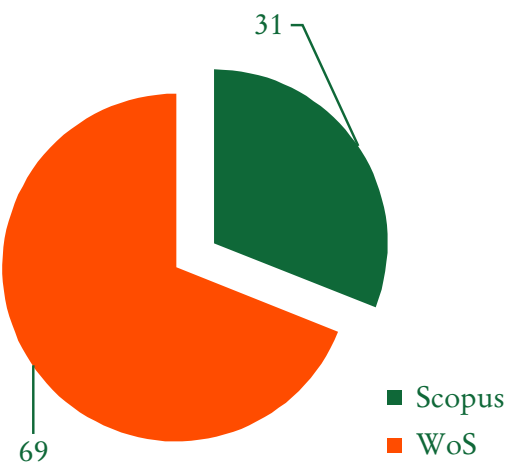

\subsection{V2. Número de registros en la producción científica desde los años 2009 a 2019}

En cuanto a la sucesión de publicaciones por año natural analizado, en la base de datos Scopus los registros han sido en el año 2018 una publicación, en el año 2017 seis publicaciones, en el año 2016 una publicación, en el año 2014 una publicación, en el año 2013 una publicación, en el año 2010 una publicación, y en el año 2009 dos publicaciones. En cambio, en la base de datos Web of Science (WoS) podemos observar que en el año 2019 hay cuatro registros, en el año 2018 hay un registro, en el año 2017

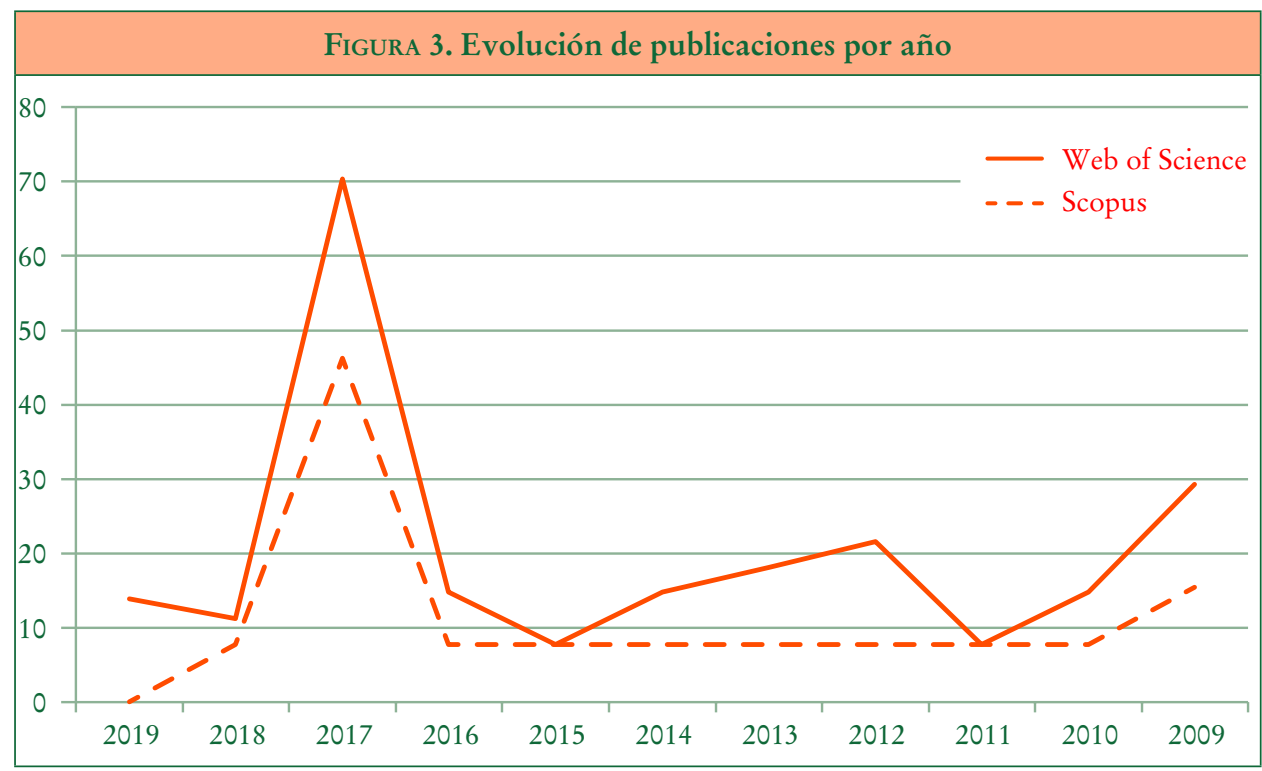

Ediciones Universidad de Salamanca / CC BY-NC-ND

Siglo Cero, vol. 51 (3), 2020, julio-septiembre, pp. 47-60 
hay siete registros, en el año 2016 hay dos registros, en el 2014 hay dos registros, en el 2013 tres registros, en el 2012 cuatro registros, en el 2010 dos registros y en el año 2009 cuatro registros. En la Figura 3 podemos ver la evolución de los porcentajes pertenecientes a cada base de datos y año.

\subsection{V3. Número de registros por tipología de documentos publicados}

Respecto al tipo de documento predominan los artículos científicos seguidos de comunicaciones y revisiones, debido a que las bases de datos que se incluyen

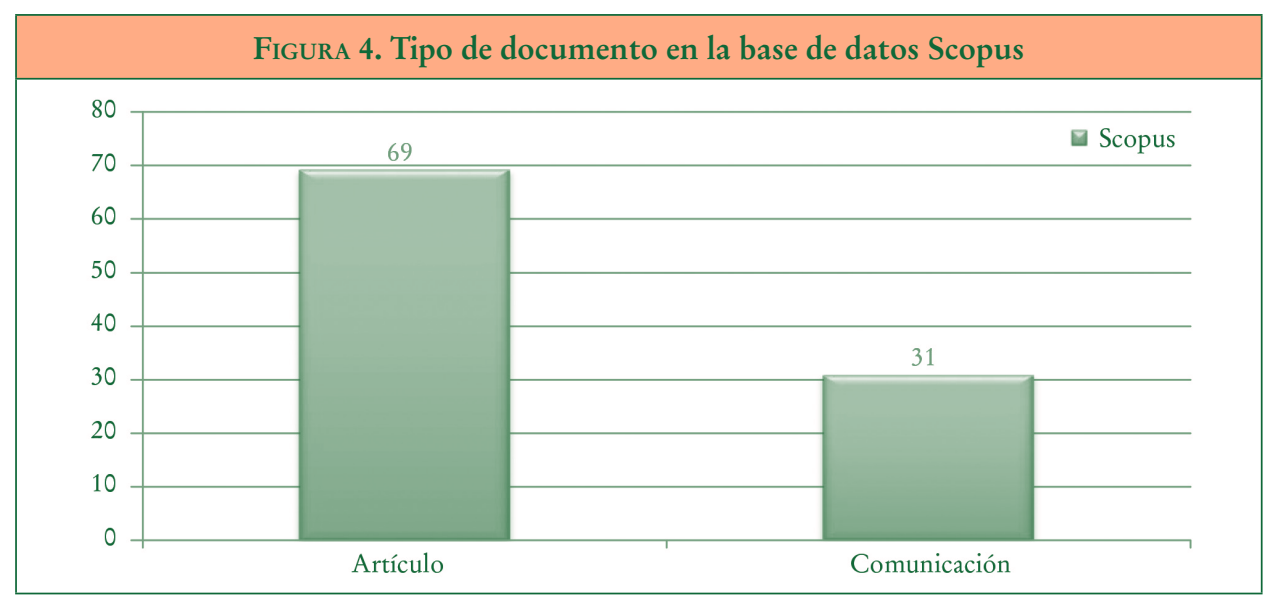

Figura 5. Tipo de documento en la base de datos Web of Science

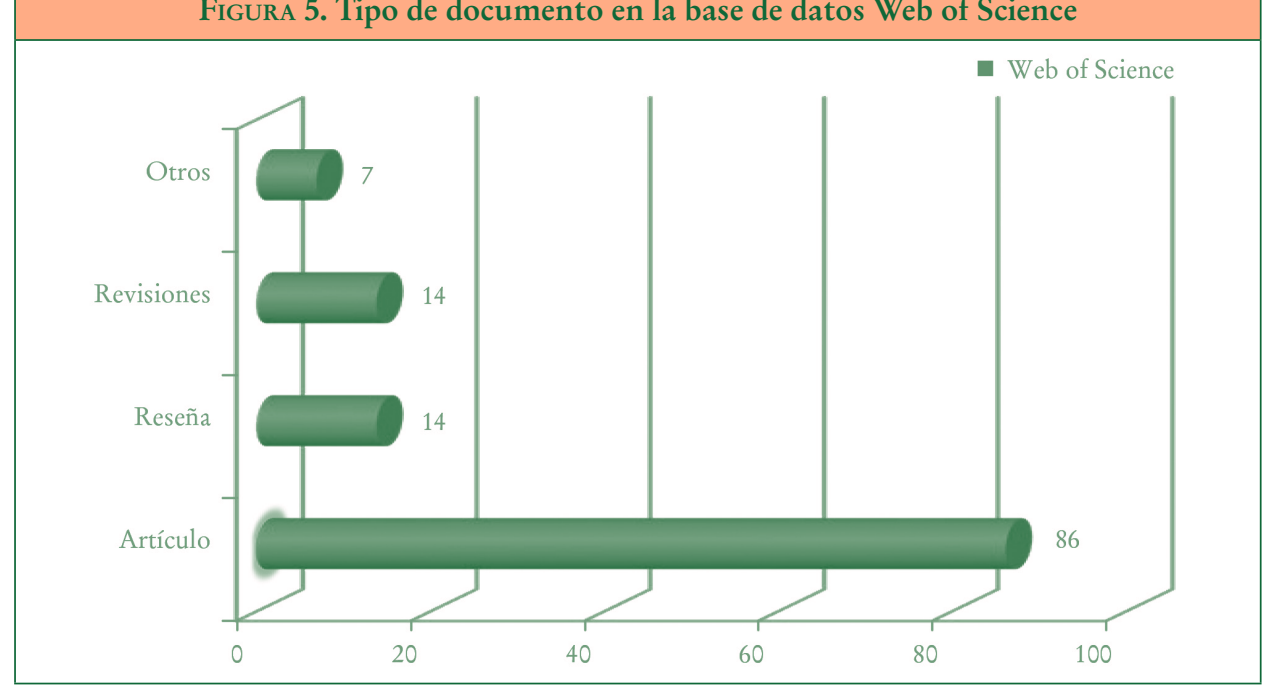

Ediciones Universidad de Salamanca / CC BY-NC-ND

Siglo Cero, vol. 51 (3), 2020, julio-septiembre, pp. 47-60

$$
-53-
$$


principalmente son revistas científicas. Atendiendo a la base de datos Scopus hay 9 artículos y 4 comunicaciones. Del mismo modo, en la base de datos Web of Science encontramos 25 artículos, 4 reseñas, 2 revisiones y 4 registros catalogados como otros. En las Figuras 4 y 5 podemos ver los porcentajes correspondientes atendiendo a la relación entre el tipo de documento publicado y la base de datos en la que lo podemos encontrar.

3.4. V4. Número de registros por titulos de las fuentes procedentes de la literatura cientifica de mayor impacto

Haciendo referencia a las revistas de mayor indexación en relación al número de registros publicados en Ciencias Sociales, en la base de datos Web of Science encontramos las revistas Journal of Applied Research in Intellectual Disabilities (59,37 \%), Journal of Intellectual Disability Research (31,25 \%) y Omega (9,38 \%). En Scopus encontramos Journal of Applied Research in Intellectual Disabilities (61,54 \%), Omega $(30,76 \%)$ y Journal of Policy and Practice in Intellectual Disabilities (7,70\%).

\section{TABLA 1. Registros de las revistas científicas de mayor impacto}

\begin{tabular}{|l|c|l|c|}
\hline \multicolumn{1}{|c|}{ Web of Science } & $\mathbf{N}$ & \multicolumn{1}{|c|}{ Scopus } & $\mathbf{N}$ \\
\hline $\begin{array}{l}\text { Journal of Applied Research in } \\
\text { Intellectual Disabilities }\end{array}$ & 19 & $\begin{array}{l}\text { Journal of Applied Research in } \\
\text { Intellectual Disabilities }\end{array}$ & 8 \\
\hline $\begin{array}{l}\text { Journal of Intellectual Disability } \\
\text { Research }\end{array}$ & 10 & Omega & 4 \\
\hline Omega & 3 & $\begin{array}{l}\text { Journal of Policy and Practice in } \\
\text { Intellectual Disabilities }\end{array}$ & 1 \\
\hline
\end{tabular}

\subsection{V5. Número de registros por países}

Estados Unidos, Irlanda, Reino Unido y España son los países que tienen más producción indexada en Scopus. Irlanda, Estados Unidos, Inglaterra, Escocia y China en la Web of Science. En la Tabla 2 podemos ver el número y el porcentaje de ambas bases de datos.

\begin{tabular}{|l|c|c|}
\hline \multicolumn{3}{|c|}{ Tabla 2. Internacionalización de la investigación } \\
\hline \multicolumn{1}{|c|}{ Scopus } & N & $\%$ \\
\hline Estados Unidos & 5 & 38,46 \\
\hline Irlanda & 4 & 30,77 \\
\hline Reino Unido & 3 & 23,08 \\
\hline España & 1 & 7,69 \\
\hline
\end{tabular}




\begin{tabular}{|l|r|r|}
\hline \multicolumn{4}{|c|}{ TABLA 2. Internacionalización de la investigación (cont.) } \\
\hline \multicolumn{1}{|c|}{ Web of Science } & N & $\%$ \\
\hline Irlanda & 15 & 51,72 \\
\hline Estados Unidos & 7 & 24,14 \\
\hline Inglaterra & 4 & 13,79 \\
\hline Escocia & 2 & 6,90 \\
\hline China & 1 & 3,45 \\
\hline
\end{tabular}

\subsection{V6. Número de registros por instituciones}

La Universidad de Oviedo, Universidad del Norte de Illinois, Universidad del Este de Washington, Instituto de Tecnología Dundalk, Fundación COPE, Federación Nacional de Organismos Voluntarios, Servicios de Discapacidad de Brothers of Charity, Universidad Nacional de Dublín, Universidad de Dundee y Trinity College Dublín son las principales instituciones de registro en la base de datos Scopus. Colegio de Salud y Ciencias Sociales de la Universidad Norte de Illinois, Esmee Fairbairn, Fondo de Desarrollo de Bienestar Social de Hong Kong, Fundación Irlandesa del Hospicio, Programa de becarios doctorales John A Hartford, Universidad de Oviedo, Instituto de Queen de Enfermeras del distrito en Irlanda y Grupo Gid en la base de datos Web os Science.

\begin{tabular}{|l|c|c|}
\hline \multicolumn{3}{|c|}{ TABLA 3. Institución de la investigación } \\
\hline \multicolumn{1}{|c|}{ Scopus } & N & $\%$ \\
\hline Oviedo University & 2 & 15,38 \\
\hline Northern Illinois University & 2 & 15,38 \\
\hline Eastern Washington University & 2 & 15,38 \\
\hline Dundalk Institute ff Technology & 1 & 7,69 \\
\hline COPE Foundation & 1 & 7,69 \\
\hline National Federation of Voluntary Bodies & 1 & 7,69 \\
\hline Brothers of Charity Disability Services & 1 & 7,69 \\
\hline University College Dublin & 1 & 7,69 \\
\hline University of Dundee & 1 & 7,69 \\
\hline Trinity College Dublin & 1 & 7,69 \\
\hline \multicolumn{1}{|c|}{ Web Of Science } & N & $\%$ \\
\hline Esmee Fairbairn & 7 & 24,14 \\
\hline Northern Illinois University College of Health and Human Sciences & 5 & 17,24 \\
\hline Hong Kong Social Welfare Development Fund & 5 & 17,24 \\
\hline Irish Hospice Foundation & 3 & 10,34 \\
\hline John A Hartford Doctoral Fellows Program & 3 & 10,34 \\
\hline Oviedo University & 2 & 6,90 \\
\hline Queen Institute of District Nurses in Ireland & 2 & 6,90 \\
\hline Grupuo Gid & 2 & 6,90 \\
\hline
\end{tabular}




\subsection{V7. Número de registros por autores}

En relación a los autores con más producción atendiendo a estos descriptores, en la base de datos Web of Science destacamos a Guerin, S. (10 registros), McEvoy, J. (9 registros), Dodd, P. (7 registros) y Clute, M. A. (3 registros), junto a los autores de la base de datos Scopus, Alcedo Rodríguez, M. Á. (2 registros), Gray, J. A. (2 registros), Gómez Sánchez, L. E. (2 registros), McEvoy, J. (2 registros), Abendroth, M. (1 registro), Arias González, V. B. (1 registro), Clute, M. (1 registros), Connaire, K. (1 registro) y Cristóbal Fernández, L. (1 registro).

\begin{tabular}{|c|c|c|}
\hline \multicolumn{3}{|c|}{ TABLA 4. Autoría } \\
\hline Web of Science & $\mathbf{N}$ & $\%$ \\
\hline Guerin, S. & 10 & 34,48 \\
\hline McEvoy, J. & 9 & 31,03 \\
\hline Dodd, P. & 7 & 24,14 \\
\hline Clute, M. A. & 3 & 10,34 \\
\hline Scopus & $\mathbf{N}$ & $\%$ \\
\hline Alcedo Rodríguez, M. Á. & 2 & 15,38 \\
\hline Gray, J. A. & 2 & 15,38 \\
\hline Gómez Sánchez, L. E. & 2 & 15,38 \\
\hline McEvoy, J. & 2 & 15,38 \\
\hline Abendroth, M. & 1 & 7,69 \\
\hline Arias González, V. B. & 1 & 7,69 \\
\hline Clute, M. & 1 & 7,69 \\
\hline Connaire, K. & 1 & 7,69 \\
\hline Cristóbal Fernández, L. & 1 & 7,69 \\
\hline
\end{tabular}

\subsection{V9. Publicaciones con mayor impacto}

Por último, en las siguientes tablas podemos ver las investigaciones de mayor impacto. En esta variable hemos tenido en cuenta los artículos con un número mínimo de citas, siendo 10 .

\begin{tabular}{|c|c|c|c|c|}
\hline \multicolumn{3}{|c|}{ Tabla 5. Artículos más citados en la Web of Science } \\
\hline \multicolumn{1}{|c|}{ Web of Science } & Autores & \multicolumn{1}{|c|}{ Revista } & Año & $\begin{array}{l}\text { Número } \\
\text { de citas }\end{array}$ \\
\hline $\begin{array}{l}\text { Concept of death and perceptions of bereave- } \\
\text { ment in adults with intellectual disabilities }\end{array}$ & $\begin{array}{l}\text { McEvoy, J., } \\
\text { MacHale, R., } \\
\text { Tierney, E. }\end{array}$ & $\begin{array}{l}\text { Journal of } \\
\text { Intellectual } \\
\text { Disability } \\
\text { Research }\end{array}$ & 2012 & 25 \\
\hline
\end{tabular}




\section{Tabla 5. Artículos más citados en la Web of Science (cont.)}

\begin{tabular}{|l|l|l|c|c|}
\hline \multicolumn{1}{|c|}{ Web of Science } & \multicolumn{1}{|c|}{ Autores } & Revista & Año & $\begin{array}{l}\text { Número } \\
\text { de citas }\end{array}$ \\
\hline $\begin{array}{l}\text { Caregiver perceptions of the understanding } \\
\text { of death and need for bereavement support in } \\
\text { adults with intellectual disabilities }\end{array}$ & $\begin{array}{l}\text { MacHale, R., } \\
\text { McEvoy, J., } \\
\text { Tierney, E. }\end{array}$ & $\begin{array}{l}\text { Journal of } \\
\text { Applied } \\
\text { Research in } \\
\text { Intellectual } \\
\text { Disabilities }\end{array}$ & 2009 & 17 \\
\hline $\begin{array}{l}\text { Grief and bereavement in people with intellec- } \\
\text { tual disabilities }\end{array}$ & $\begin{array}{l}\text { Dodd, P., } \\
\text { Guerin, S. }\end{array}$ & $\begin{array}{l}\text { Current } \\
\text { Opinion in } \\
\text { Psychiatry }\end{array}$ & 2009 & 15 \\
\hline $\begin{array}{l}\text { The experience of carers in supporting people } \\
\text { with intellectual disabilities through the process } \\
\text { of bereavement: an interpretative phenomeno- } \\
\text { logical analysis }\end{array}$ & $\begin{array}{l}\text { Handley, E., } \\
\text { Hutchinson, }\end{array}$ & $\begin{array}{l}\text { Journal of } \\
\text { Applied } \\
\text { Research in } \\
\text { Intellectual } \\
\text { Disabilities }\end{array}$ & 2013 & 13 \\
\hline $\begin{array}{l}\text { Supporting adults with an intellectual disability } \\
\text { during experiences of loss and bereavement: staff } \\
\text { views, experiences and suggestions for training }\end{array}$ & $\begin{array}{l}\text { McEvoy, J., } \\
\text { Guerin, S., } \\
\text { Dodd, P., } \\
\text { Hillery, J. }\end{array}$ & $\begin{array}{l}\text { Journal of } \\
\text { Applied } \\
\text { Research in } \\
\text { Intellectual } \\
\text { Disabilities }\end{array}$ & 2010 & 13 \\
\hline $\begin{array}{l}\text { An initial assessment of the psychometric proper- } \\
\text { ties of the Complicated Grief Questionnaire for } \\
\text { People with Intellectual Disabilities (CGQ-ID) }\end{array}$ & $\begin{array}{l}\text { Guerin, S., } \\
\text { Dodd, P., } \\
\text { Tyrell, J., } \\
\text { McEvoy, J., } \\
\text { Buckley, S., } \\
\text { Hillery, J. }\end{array}$ & $\begin{array}{l}\text { Research in } \\
\text { Developmental } \\
\text { Disabilities }\end{array}$ & 2009 & 11 \\
\hline
\end{tabular}

\section{Tabla 6. Artículos más citados en Scopus}

\begin{tabular}{|l|l|l|c|c|}
\hline \multicolumn{1}{|c|}{ Título } & \multicolumn{1}{|c|}{ Autores } & \multicolumn{1}{c|}{ Revista } & Año & $\begin{array}{c}\text { Número } \\
\text { de citas }\end{array}$ \\
\hline $\begin{array}{l}\text { Developing an education intervention } \\
\text { for staff supporting persons with an } \\
\text { intellectual disability and advanced } \\
\text { dementia }\end{array}$ & $\begin{array}{l}\text { Fahey-McCarthy, } \\
\text { E., McCarron, } \\
\text { M., Connaire, K., } \\
\text { McCallion, P. }\end{array}$ & $\begin{array}{l}\text { Journal of Policy } \\
\text { and Practice } \\
\text { in Intellectual } \\
\text { Disabilities }\end{array}$ & 2009 & 27 \\
\hline $\begin{array}{l}\text { Caregiver perceptions of the } \\
\text { understanding of death and need for } \\
\text { bereavement support in adults with } \\
\text { intellectual disabilities }\end{array}$ & $\begin{array}{l}\text { MacHale, R., } \\
\text { McEvoy, J., } \\
\text { Tierney, E. }\end{array}$ & $\begin{array}{l}\text { Journal of } \\
\text { Applied Research } \\
\text { in Intellectual } \\
\text { Disabilities }\end{array}$ & 2009 & 17 \\
\hline $\begin{array}{l}\text { The experience of carers in supporting } \\
\text { people with intellectual disabilities } \\
\text { through the process of bereavement: } \\
\text { an interpretative phenomenological } \\
\text { analysis }\end{array}$ & $\begin{array}{l}\text { Handley, E., } \\
\text { Hutchinson, N. }\end{array}$ & $\begin{array}{l}\text { Journal of } \\
\text { Applied Research } \\
\text { in Intellectual } \\
\text { Disabilities }\end{array}$ & 2013 & 12 \\
\hline
\end{tabular}




\section{Discusión y conclusiones}

La realización de este estudio ha puesto de manifiesto que la investigación del duelo en personas con discapacidad intelectual es aún muy incipiente; por esta razón, con el propósito de conocer las últimas investigaciones realizadas en este campo, se ha propuesto el siguiente objetivo de investigación: analizar cienciométricamente la producción científica de mayor impacto sobre el duelo y la pérdida en personas con discapacidad intelectual. Se ha realizado una revisión cienciométrica de estudios bibliográficos, cuantificando las variables en relación a tres descriptores "bereavement", “intellectual disability" y "grief” de las publicaciones de los últimos diez años en las colecciones principales de las bases de datos Scopus y Web of Science (Wos). La muestra final de referencias ha sido de 42, de las cuales 13 pertenecen a la base de datos Scopus y 29 a la base de datos Web of Science. Los resultados obtenidos poseen especial relevancia ya que han permitido hacer visible la investigación realizada en este campo estando todavía en vías de desarrollo, resaltado los estudios realizados por Fernández-Ávalos, Fernández-Alcántara, Cruz-Quintana y Pérez-Marfil (2017) y Domingo, Cuesta y Sánchez (2018), en el que exponen que la principal limitación de sus estudios ha sido la escasez de investigaciones realizadas y la escasa bibliografía reciente sobre el proceso de duelo en personas con discapacidad intelectual.

Algunas de estas investigaciones ponen de manifiesto que en la mayoría de los casos la información se basa en la proporcionada por los cuidadores, señalando que son pocos los estudios que recogen información de las personas con discapacidad intelectual, con muestras poco amplias y muy heterogéneas, para poder realizar conclusiones más amplias. Por otro lado, no existe un protocolo de actuación ante el duelo en personas con discapacidad intelectual, aunque existen ciertas guías que pueden ayudar a la persona a sobrellevar la pérdida y a los profesionales a tener herramientas que favorezcan el apoyo. Para finalizar, ponemos de manifiesto la necesidad de seguir trabajando sobre este tema, en una misma línea y de manera individualizada, ya que cada persona requiere de unas estrategias educativas diferentes atendiendo a las características personales. Es necesario trabajar en el desarrollo de investigaciones para conocer de manera detallada las características en el proceso de esta población, junto a una herramienta de uso profesional que ayude a determinar las necesidades de apoyo de las personas con discapacidad intelectual que pasan por estos procesos de duelo para poder intervenir desarrollando prácticas basadas en evidencias (Fernández, Alcedo y Gómez, 2018).

La principal limitación del presente estudio se centra en la escasez de investigaciones realizadas sobre el duelo en personas con discapacidad intelectual; del mismo modo, la literatura científica acerca del proceso de duelo, la diversidad y sus síntomas, así como las experiencias específicas en personas con DI, son escasos. Finalmente, se resalta como línea de investigación futura analizar de manera bibliométrica las tesis doctorales en relación a la temática del artículo o una revisión cualitativa de los artículos analizados cuantitativamente en este estudio. 


\section{Referencias bibliográficas}

Bild, R. y Gómez, I. (2008). Acompañamiento creativo. Metodología para el cuidado del niño y adolescente con enfermedad terminal y su familia. Valencia: Conselleria de Sanitat.

Blackman, N. (2016). Supporting people with learning disabilities through a bereavement. Tizard Learning Disability Review, 21, 199-202.

Brickell, C. y Munir, K. (2008). La pena y sus complicaciones en personas con discapacidad intelectual. Harvard Review of Psychiatry, 16, 1-12.

Bucay, J. (2006). El camino de las lágrimas. México: Editorial Sudamericana, Del Nuevo Extremo.

Campbell, A. y Bell, D. (2010). Sad, just sad: a woman with a learning disability experiencing bereavement. British Journal of Learning Disabilities, 39, 11-16.

Corrales-Reyes, I. E., Fornaris-Cedeno, Y. y Reyes-Pérez, J. J. (2018). Análisis bibliométrico de la revista Investigación en Educación Médica. Período 2012-2016. Investigación en Educación Médica, 7(25), 18-26.

Domingo, E., Cuesta, J. L. y Sánchez, S. (2018). Cómo afrontar el duelo en las personas con discapacidad. Una aproximación al problema. Siglo Cero, 49(4), 51-68.

Fernández, L., Alcedo, M. A. y Gómez, L. (2018). Duelo en discapacidad intelectual: los avances de una década. Revista Española de Discapacidad (REDIS), 5(2), 53-72.

Fernández-Avalos, I. M., Fernández-Alcántara, M., Cruz-Quintana, F. y Pérez-MarFIL, M. N. (2017). Assessment of the grieving processes in people with intellectual disabilities: a systematic review. Estudios de Psicología, 38(3), 779-787.

Fuentes, A. R. y Ortega, J. L. G. (2019). Análisis bibliométrico sobre educación especial. Profesorado, Revista de Currículum y Formación del Profesorado, 23(1), 307-327.

González Romero, I. y López, L. (2013). Proceso de duelo, estrategias de afrontamiento y resiliencia en adultos ante la muerte de un ser querido (trabajo fin de grado). Escuela de Psicología: Maracaibo.

James, I. A. (1995). Helping people with learning disability to cope with bereavement. British Journal of Learning Disabilities, 23, 74-78.

McRitchie, R., McKenzie, K., Quayle, E., Harlin, M. y Neumann, K. (2014). How adults with an intellectual disability experience bereavement and grief: a qualitative exploration. Death studies, 38(3), 179-185.

Moorley, C. R., Cahill, S. y Corcoran, N. T. (2016). Life after Stroke: coping mechanisms among African Caribbean Women. Health y Social Care in the Community, 24(6), 769-778.

Muñız, V., Alcedo, M. Á. y Gómez, L. E. (2017). El proceso de duelo de personas con discapacidad intelectual. Siglo Cero, 48(3), 7-25.

Pertegal-Vega, M. A., Oliva-Delgado, A. y Rodríguez-Meirinhos, A. (2019). Systematic review of the current state of research on Online Social Networks: taxonomy on experience of use. [Revisión sistemática del panorama de la investigación sobre redes sociales: Taxonomía sobre experiencias de uso]. Comunicar, 60, 81-91.

Poch, C. y Herrero, O. (2003). La muerte y el duelo en el contexto educativo. Reflexiones, testimonios y actividades. Barcelona: Paidós.

Robles, J. I. y Medina, J. L (2002). Intervención psicológica en las catástrofes. Madrid: Síntesis. Rodríguez, A. y Gallego, J. L. (2019). Análisis bibliométrico sobre educación especial. Profesorado. Revista de Currículum y Formación de Profesorado, 23(1), 307-327.

Rodríguez Herrero, P., Izuzquiza, D. y Herrán, A. (2015). Concept of death in young people with intellectual disability: a contribution to the pedagogy on death. Siglo Cero, 46(1), 67-80. 
Salgado-Orellana, N., Díaz-Levicoy, D., Ferrada, C. y Parraguez, R. (2019). La investigación sobre acción tutorial en España: un análisis cienciométrico en las tesis de doctorado entre 1980 y 2016. Revista de Orientación Educacional, 33(63), 63-81.

Shear, M. K. (2012). Getting straight about grief. Depression and Anxiety, 29(6), 461-464.

Solana, C. A. (2005). Aspectos psicológicos en el paciente superviviente. Oncología, 28(3), 51-57.

Worden, W. (2010). El tratamiento del duelo: asesoramiento psicológico y terapia. Barcelona: Paidós.

Ediciones Universidad de Salamanca / CC BY-NC-ND

Siglo Cero, vol. 51 (3), 2020, julio-septiembre, pp. 47-60

$$
-60-
$$

